\title{
LESSONS FROM NATURE IN RESOLVING INTERFACE PROBLEMS IN DESIGN OF STRUCTURES AND SYSTEMS
}

\author{
L.D. VASSILEVA \\ Department of Civil Engineering, University of Zimbabwe, Zimbabwe
}

\begin{abstract}
Design prototypes for the physical and social worlds are found in nature in the domains of flora, fauna and the human body. However, in designing and developing structures and systems, learning from the common creative principles in nature is probably more important than borrowing formal patterns and models. Physical structures and systems in development are designed and modified in response to needs that arise at various interfaces concerning societies and individuals. Principles, learned from nature, and adopted as approaches to structural and behavior aspects of design can assist in resolving successfully intrinsic and extrinsic interface problems of interactions in space and time. The significance of the principles of heterogeneity, complexity, adaptability and integration grows with ever-deepening exploration of nature. Their manifestations are found in an endless variety of forms and relationships in the universe. The lessons derived from nature contribute to a general philosophy of engineering design and justify solutions toward innovations and sustainable existence.

Keywords: adaptability, complexity, heterogeneity, integration.
\end{abstract}

\section{INTRODUCTION}

Since ancient times, nature has provided man with inspirations, knowledge and models for application in designing systems, structures and their constituent parts. Nature, as related to evolution and development, creates an endless variety of complex forms and interactive functions. The natural richness of flora, fauna and the human body offers an abundance of prototypes for the physical and social world.

The dynamics of nature, as transmitted into a developing material and social world, opens multiple opportunities for innovative creations of physical forms and abstract systems. Current exploration of the universe, added to past experience and knowledge, widens the possibilities of further learning from nature. While formal similarities and prototypes are useful, the understanding of general common principles in nature can be even more valuable in cognitive and behavioral aspects of design. These principles could explain internal and external interactions within, as well as between various structures, systems and their environments [1].

In this context, any complex whole constituted of many interrelated elements would represent a structure, while an enveloping group of physical wholes or arrangement principles may be considered a system, irrespective of categories, types and sizes.

The advancement in the understanding of nature confirms the existence and significance of principles that could be recognized as universally attributable to design. With the aim to review and emphasize on the important, possible and useful nature-based approaches in resolving problems of interaction in space and time, the key principles, which may be recognized in nature, are here discussed. These distinct principles are heterogeneity, complexity, adaptability and integration.

Within the vast interplay of factors that affect design in material environments, and in particular engineering design, the clue toward sustainable existence is resolving interface problems. Generally, sustainability could be defined as a series of beneficial interactions in space and time in both the natural and the man-made world. As per universal definition, interface problems arise when two or more subjects or their parts meet, co-exist and affect each other. From the engineering point of view, 
interface problems arise at common surfaces where there may occur or are found various transitions of data, physical flows, circuits, etc. from one to another system or structure.

Tentatively, the general classes that could be considered are interface problems within individual structures and systems, those between structures/systems of similar categories and interface problems between those of different categories. The terms "structure" and "system," as defined previously, are used for any item or grouping of items that are subjects of design for existence. Studies and analysis of nature show that the above-mentioned principles lead to interactions at interfaces. Therefore, the principles found in the natural processes may be considered useful general concepts in evolutionary science, which assist in understanding the process of design. These principles appear to be applicable, desirable and even unavoidable and particularly valid for engineering and architectural design.

\section{HETEROGENEITY}

Heterogeneity is in the foundations of all systems and structures, regardless of the nature [2]. Any structure is a complex whole that is built or made from interacting parts put together. Any system is a group of items (structures, subsystems) organized in a particular manner to work together as a whole due to interactions. Herein, the terms "structure" and "system" could be used interchangeably.

Heterogeneity of relevant parameters as a limited or large variety of substances and compositions is the fundamental notion for all natural things in relation to the flora, fauna and the human body. It implies diversity of building cells or units and functional interrelationships. The spatial and temporal interactions at various levels of relationships within a given system affect some, if not all, of its parts, as well as its totality. For instance, cancerous cells in a living body destroy some organs and in time affect the entire organism. Whatever could be the arising interface problems in similar situations, the potential solutions are always interpretations of three possible outcomes, which could be either destruction, protection or a mixture of both.

Similarly, any process of conscious design, carried out by man, begins with an inventory of existing and/or desired elements of heterogeneity. The levels of heterogeneity would depend on typologies of cells/units and their internal relationships and would determine different levels of complexity. An analysis of heterogeneity is the initial necessary step in resolving interface problems within a single system, as well as between two and more systems. In nature, it is mostly linked to settings and situations. In the physical world designed by man, it entails a necessary selection of relevant expectations and needs, based on past experience and current knowledge.

\subsection{Destruction}

In the course of natural evolution, destructive tendencies toward solving interface problems of spatial and temporal manifestations are probably the oldest outcomes defined by man. Animal and plant species have disappeared, and probably continue changing, and long selective processes establish a relatively sustainable flora and fauna. Some built-in characteristics of heterogeneity lead to processes of selection and survival.

A destructive approach to resolving interface problems could be either total or partial. Species in nature disappear as a result of total but not desultory destructions, because some remainders happen to be adaptable due to compatible structural, behavioral and functional re-arrangements, which have temporal, mostly long-term, manifestations. In partial destructions, stability of some structural members and layers of a system or systems are usually tested through various internal and external energies of interactivity. 


\subsection{Protection}

Another possible outcome based on heterogeneity is protection, which could be partial or a structural wholeness, most often the former. As a principal approach to natural interface problems, protection is very closely related to sustainability, although protected systems and structures are not exactly unchanged indefinitely in time and space.

Protection is usually embedded in some purposely-created features in order to sustain a longer life. Despite an abundance of features in animal and plant kingdom, such as protective coloring, poison substances, specifically developed members like particularly shaped tongues, etc., an absolute wholeness in protection is not often observed. Some changes may occur in major or in secondary features in the natural world as adaptable mixtures of destruction and protection.

In man-made designs, widely used approaches, as lessons from nature, comprise both destruction and protection in resolving interface problems, for example, adjustments and modifications in the architectural and urban practice and in all engineering fields of design. The two approaches weave in particular ways according to the specific complexities of individual cases.

\section{COMPLEXITY}

Complexity is a common creative building principle and attribute of nature, thus including all kinds of systems and structures from the highest types of compositions in descending orders down to the simplest cells or units. In general, the complexity of any system or structure can be defined as made up of various interconnected units/parts or cells/particles, which are tied up and linked by specific relationships that sometimes are difficult to understand.

A full definition of complexity should contain explanations about the functional relationships and the manners and continuity of interactions between the constituent parts. The degrees of complexity may vary widely depending on the numbers and types of cells/parts, functions and durations of internal and external interactions.

Detailed studies of different natural systems can reveal general rules of major importance that pertain to the building and governing of their complexity, as confirmed by Ciofalo et al. [3] and Tiezzi [4]. These rules can be found behind the endless variety of relationships in all domains of nature, for example the genes in all living organisms.

At least three major modes or rules can explain internal and external interactions in relation to the complexity of nature and within natural environments and these are coordination, subordination and hierarchy.

Similar governing rules could be used in the man-made world in designing complex physical, environmental and social systems, where the patterns may comprise a large variety of non-uniform groups of parts and arrangements [5]. While coordination is typically the principle found in all degrees of complexity, the principles of subordination and hierarchy may not be always present.

\subsection{Coordination}

Coordination is the most essential mode of interactive relationships in creating complexity and making different cells/parts and groups of parts to function purposely and efficiently together, as shown by Masters [1] and recently by Schoeman [6].

This is supported by many, some relatively simple, examples of flora and fauna in their natural environments, as well as by the most complex relationship in the central nervous system of the human body. 
The principle of coordination is fundamentally linked with an ability of controlling the functioning of a system or structure, for example, the role of Alfa-males in feeding new born in the hierarchical social structures of the gray wolfs.

The manner of individual complexity usually determines the ability of structures or systems to react to disturbed equilibriums by changes in substance or function. Broadly speaking, natural reactions to broken equilibriums between species and environments comprise partial or complete destructions within long periods of time, or adaptations for renewal.

Similar reactions are adopted in the man-made world through design, where conscious creativity is aimed to bring about a balance between demand and supply, as in physical and environmental structures and systems of various functions.

\subsection{Hierarchy}

A common and well-pronounced mode of building complexity in nature is the hierarchical arrangement, where particular systems in terms of status and authority of interlinked levels determine and control interactions of the constituent parts [7].

The principle of hierarchy is found in naturally existing flora and fauna and has been scientifically proved by man. It is found in general classifications of species, in their social group structures and in their individual organs and parts. It is a common principle of interactive relationships in the human body as well. The hierarchical relationships contribute to a large variety of degrees and orders of complexity from a straight-line dependence to groupings with specific links by typical attributes and continuity. Their complex sequences are related to the fundamental heterogeneity of constituent cells and functions.

As an example, the blood-circulating systems of animals and of the human body are structured in a hierarchical manner, where branches of lower ranks are linked to higher-rank vessels. At the top of this strongly emphasized hierarchy is the pump, which controls the distribution, and that is the heart.

Many man-made models imitate to a large extent some of these models of hierarchical relationships and structural schemes in nature, for example, artificial models of management.

\subsection{Subordination}

Subordination is also an essential principal mode in building complexity, which supports hierarchical structures. It could be defined as a manner of use and treatment of basic cells or units and their possible groupings within an individual hierarchical system and in conjunction with the assigned functional relationships. As a structural arrangement and as a process, subordination depends on a natural heterogeneity and on internal and external links of interactivity [8], such as flows of distribution that form the complexity of a system.

Nature shows that less heterogeneous structures, built by small numbers of different cell or unit types, have much simpler hierarchical patterns, but relationships of higher complex orders of subordination control the functional efficiency of the entity. For instance, two major types of blood cells in the living body (e.g., white and red, or good and bad) use the complex system of a higher order of blood distribution.

\section{ADAPTABILITY}

Adaptability is a powerful ability of some systems and parts thereof to adjust and become suitable to possible new uses, changed situations and circumstances. It is a principal and temporal attribute of 
the natural world in relation to its heterogeneity and complexity [5, 6, 9-11]. This power of adapting explains the sustained existence of species [7] and the occurrence of new and disappearance of transient species throughout natural evolution. It is adaptation to environmental conditions that explains some particular features typical of species - that some species of trees lose their leaves in winter, others are evergreens or cactuses have special types of leaves.

In building the physical world during periods of historical development, human societies interpret this fundamental principle of nature in multifarious ways according to spatial and temporal needs and circumstances. In civil engineering and architecture, projects must be adapted to natural and man-made environments. The best orientation of habitable rooms in the northern hemisphere is facing south, while in the southern hemisphere it is north. In southern countries the windows are larger than those in northern countries. Where two-directional vehicular flows reach capacity limits of some streets, engineers try to adapt and manage traffic $[6,12]$ as one-way operations or, alternatively, reduce traffic volumes by redirecting flows over renewed street networks.

As nature proves, there are two principal modes concerning adaptability of systems, structures and parts thereof - suitability and compatibility. These modes apply to two major cases.

First, patterns of adapted behavior are sustained due to complex and relatively stable relationships with the environment, as with seasonally falling leaves.

Second, justified by circumstances some adaptations can arise, as, for example, in an occasional plant symbiosis or in long-term adaptations of wild animals to arctic conditions.

Adaptable cells or units, parts, groupings and systems are suitable for changed or for some new needs and can function compatibly with other contents and environments.

\subsection{Suitability}

Suitability can be defined as being right and appropriate for particular purposes, conditions and circumstances. Natural properties of matter and living creatures in the universe show that suitability features are embedded in cells/units, parts and bodies in relatively stable fundamental links, as shown by $\mathrm{Wu}$ et al. [13]. Some may as well provide, but not necessarily, for dynamic interactions that can lead to further adaptations in space and time.

Most plants are adapted to particular types of climates and soils, but just a few may grow successfully in any climatic and soil conditions. Suitability may depend upon specific criteria according to the availability of substances, structures, purposes and circumstances, as well as on compositions of interrelated internal and external characteristics, such as temperature, altitude, etc. Similarly, in engineering the suitability of some structural designs is supported by particular soils.

It is known that the bound carbohydrates found in starchy food plants such as wheat, potato or rice play special roles in nature. In different geographic locations these internal characteristics may affect suitability. Naturally or by human intervention changes can occur in conjunction [14]. This lesson, which is derived from nature, is extremely useful in human activities, in particular in design, and cannot be overemphasized.

\subsection{Compatibility}

Compatibility may be regarded at once as an ability of cells or units, parts and groupings or entire organisms to match with internal and external matter, functions and conditions and work for current purposes without becoming negatively susceptible to changes. Compatibility is not a prerequisite for assessing a system or structure as being adaptable to internal and external changes for an extended existence. 
Examples from large ecological systems to stem cells show that compatibility is a principle of paramount importance in the natural evolutionary process. Typically, some cells or units and parts may remain unchanged, but others can change while remaining still compatible with both the original and the new purposes and conditions. A polar bear is still a bear but with white fur. The anatomy is almost the same, but cells and functions adapt to the harsh and quite different polar conditions.

Similarly, in man-made designs, compatibility is concerned with factors of internal and external interactions that vary widely. In architecture and urban development, conversion of warehouses into houses and some odd examples of churches into youth centers shows that compatibility does not include only the soundness of the existing structures. Features that are considered compatible to new needs and environments may include combinations of internal and external criteria, such as size, shape, height, light, space plans, materials, appearance, etc. These physical features are always accompanied by interactive relationships.

\subsection{Flexibility}

Some flora and fauna species, including their parts or cells, have adapted, adjusted to changing circumstances, while others have remained resistant. An ability to change over time in order to adapt to some changed conditions, but still be capable of working in its original form, is usually defined as flexibility. It plays a significant role in the natural evolutionary process.

Flexibility of substances, matter or species cannot be assessed without considering some particular features of suitability and compatibility in order to be adaptable. For example, natural carbohydrates, such as cellulose, starch, glycogen and most sugars, exhibit variations in constituents and linkages and are susceptible to inducible changes [14]. Similarly, a large flexibly designed hall can have inducible changes for various functions - meetings, exhibitions, etc.

Flexibility is always specifically defined. A specific cell is matching to and appropriate for its assigned functions and can coexist with other cells. Cells or genes that can bend, break or change through continuous interactions are flexible.

\section{INTEGRATION}

In natural evolution, systems reach levels of integration at which they are mature and, beyond any doubt, appropriate and effective for the assigned functions within the respective environments. Integrity in nature is a state of wholeness, of undivided and purposely created systems that are built in various models based on the principles of heterogeneity, complexity and adaptability. Natural levels of integration are never absolute. They have temporal validities of particular durations, which determine periods of sustainable existence of structures and systems within interlinked chains of evolutionary activities.

Probably, there is no better example of integration in nature than the functioning of the human brain. The processing of information in the cerebral cortex involves activation of millions of neurons, which are distributed over various areas. The activity patterns are integrated to generate coherent percepts [15]. The correlation and synchronization among widely separated cortical regions continue to be subjects of important studies for human activities and the society.

Occurrences of privations of different nature can disable the integrity of natural systems. They can cease to function as to their "original designs." Extrinsic forces and internal mutations may disrupt established patterns of behavior and misbalance equilibriums, as for example, mutations in plants caused by radiation or by global warming. Similar situations under continual interactions stimulate changes in built environments and they may needmodifications. Modifications are temporized adaptations and adjustments to changed and/or to new conditions. For some systems and structures the achieved temporal integrations may become intrinsic motivations for further changes. 


\section{LESSONS FROM NATURE}

Sustainable existence, which is effective and beneficial, is supported by developmentally established patterns of interactivity between systems and structures. The life cycles of all living species in nature are based on multitudes of heterogeneity, complexity and adaptability features that are involved in multifarious interactivities. Interactivities at various layers, scales and time frames constitute the essence of natural life [11] as well as in business life [16]. There are species that are dying, some new species are born and others are changing and continue living. Interactivity is the key to sustainable life and motivates both protection and change of structures and systems.

Based on interactivity, the intrinsic and extrinsic interface problems in nature stimulate consequential changes, which become natural solutions to these problems. In these processes, heterogeneity and complexity, adaptability and integration have common fields of interactivity and become the principles of natural creative forces in evolution.

Nature undoubtedly shows tendencies of systems and/or structures to combine, act and live together because of a certain affinity with mutual exchange of information, similarity and transitions of common or supplementary material characteristics, and functional properties that are fundamental for resolving interface problems.

The greatest importance, and unsurpassable value of nature for man-made design, is contained in the fundamental principles of creating structures and systems and of building their properties that are determined by abundances of links, shapes and relationships within particular environmental and functional frameworks. In other words, nature as a whole is a sustained and endless source of lessons in design for solving interface problems. In nature, interface problems can be encountered within single systems, as well as between two and more systems.

An attempt to illustrate these fundamental processes is made in Fig. 1. It shows schematically that an adaptability status is defined by interactive overlapping of heterogeneity and complexity features, the numbers of which do not matter. Consequently, the processes of integration provide for interface solutions in the common fields of interactivity.

The three fundamental ranges of interface problems involving human participation that could be identified are the interfaces between natural species and their environments, the interfaces between man-made systems and natural environments, and those between man-made systems and man-made environments. It should be noted that men might not affect or even know some complex interfaces within their enveloping ecosystems.

\section{CONCLUSION}

Scientific advances at the beginning of the 21st century have opened many new unforeseen prospects in human activities on an individual and social basis that require innovative and technologically new approaches to physical and social designs. As always, nature is a constant source of inspiration, with a plethora of models and patterns to follow.

Considering the ever-growing knowledge about nature and new discoveries in various geographic locations, one usually thinks that nature primarily offers new models and patterns for man-made designs. This is true to a large extent, but does not exhaust the role of nature for design. Particularly important is the knowledge about the structures and behaviors of living matter.

The philosophy of design arises from this knowledge of nature, irrespective of the individual structures or systems and their particular situations. The search for knowledge and the understanding of nature justify common scientific methods, which are applicable in design and in their interactions "form" the methodology for design.

The ultimate goal of design is solving interface problems by creating projects of integrity, sufficiently "strong" and "healthy" to be accepted and approved by their direct users and other indirect 


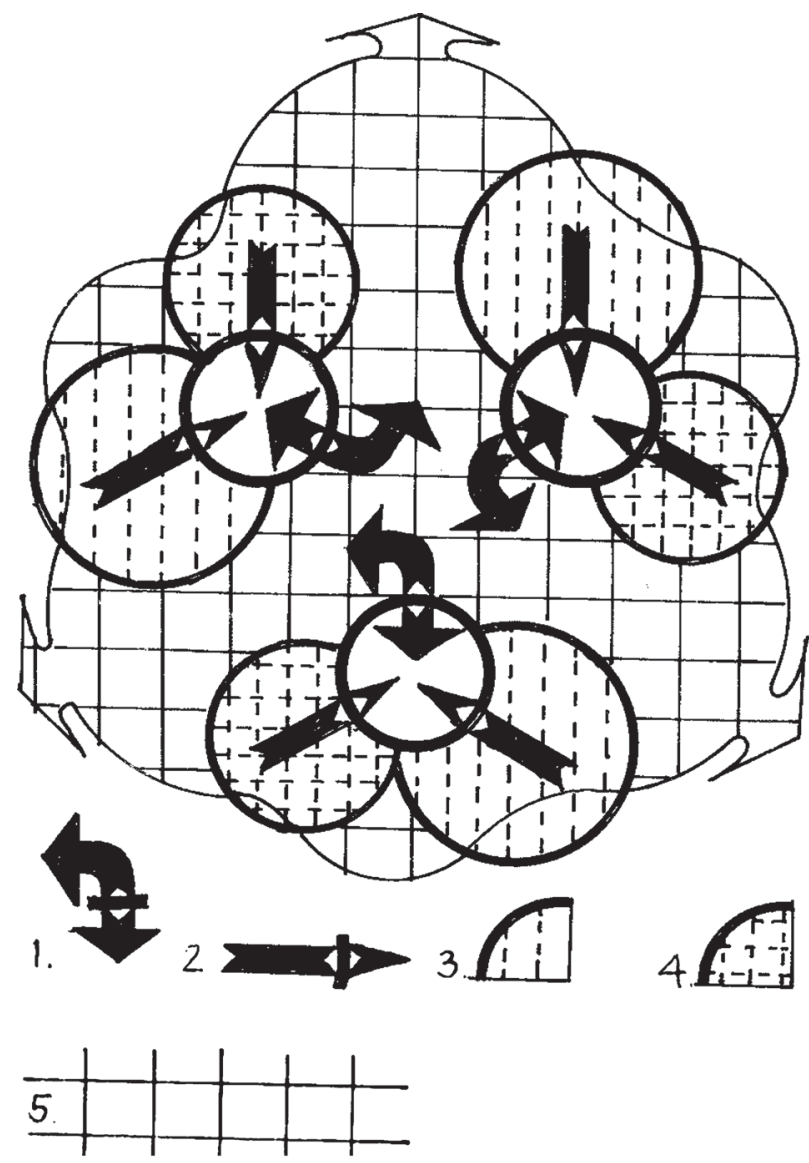

Figure 1: Fundamental principles and relationships: 1, integrity; 2, adaptability; 3, heterogeneity; 4 , complexity; 5 , field of interactivity.

interested parties, projects that would carry minimal operational risks and still remain open to possible changes. Envisaged further interface problems might ensure continuity of development and stimulate further solutions for adjustments.

Design begins with creating an inventory of heterogeneity features and relevant standards in order to capture as widely as possible data about all concerned categories. These include geographic, material, structural, esthetic and behavioral aspects, which are expected to justify applications of the principles of protection, or destruction and subversion, or alternatively, some mixed approaches.

The following step in design sets out a framework for complexity. The objectives of design, together with the need to meet relevant regulatory, statutory and esthetic requirements, lead to typical or desired arrangements and interlinks that create complexity. Complexity is defined by the applications of the principles of coordination, hierarchy and subordination.

In order to achieve operational efficiency and to sustain consistent standards, the designed structures or systems should be flexible to changes. Similar to the dynamics in nature [3], the dynamics to be considered in designing should be translated into adaptability. Therefore, defining the parameters of adaptability is of paramount importance so as to ensure that the proposed designs would be sustainable. 




Figure 2: Philosophy of design.

The energy to adapt depends on the suitability, compatibility and flexibility of constituting cells or units and groupings, on their links and relationships within the whole and between the parts thereof.

This understanding of design is concerned with interface problems in continuity, because in a dynamically evolving process an ideal solution would be a design, which can be easily adaptable and upgradeable to meet some changing requirements and circumstances [1]. Since man-made design is always aimed at resolving interface problems, appropriately proposed integrated, flexible and measurable solutions can bring about fundamental improvements and innovations in the quality of design of structures and systems.

On the other hand, design is a highly individualized process. A designer or an engineer may begin the process from various considerations depending on the scope, objective or situation. There is no single experience-proven sequence of approaches and optional choices of deployment style could arise. This is why the fundamental, major principles in design are of great importance.

The diagram presented in Fig. 2 shows the steps in the philosophy of design. The steps are summarized by considering and including the objectives, the possible approaches and the expected outcomes of 
activities. All these are confirmed, as well as being confirmed, by studies and discoveries about the physical world, natural laws and relevant societies.

In addition to an endless variety of patterns and models that might offer innovative concepts, the fundamental and guiding principles of design, derived from nature, discussed here should probably be considered as the greatest lesson of nature. This is the most essential contribution of nature to the physical and social sciences. And the widening and deepening of this knowledge supports human creativity and understanding of intended usefulness, sustainability and beauty, which altogether make the essence of design.

\section{REFERENCES}

[1] Masters, G.M., Introduction to Environmental Engineering and Science, Prentice Hall: New Jersey, 1991.

[2] Kumar, S., Lessons from Nature: The Benefits of Embryology, University College: London, 2001.

[3] Ciofalo, M., Collins, M.W. \& Hennessy, T.R., Nanoscale Fluid Dynamics in Physiological Processes, Series: Advances in Computational Bioengineering, Vol. 2, WIT Press: Southampton, 1999.

[4] Tiezzi, E., Steps Towards an Evolutionary Physics, Series: The Sustainable World, Vol. 15, WIT Press: Southampton, 2005.

[5] Coppens, M.O., Learning from Nature in Building Sustainable Chemical Technology, Design and Nature II, WIT Press: Southampton, 2000.

[6] Schoeman, C.B., The alignment between integrated transport plans and the preparation of integrated development plans in South Africa. Urban Transport X, The Built Environment, Vol. 75, 2004.

[7] Bentley, P.J. \& Kumar, S., Three ways to grow design: a comparison of embryogenesis for an evolutionary design problem. Genetic and Evolutionary Computation Conference, 1999.

[8] Rosson, B.T., Structural analysis using nature golden proportion. Design and Nature II, WIT Press: Southampton, 2004.

[9] Wolpert, L., Principles of Development, Oxford University Press, 1998.

[10] Hohl, W., Neutral networks and information interchange in building. Design and Nature II, WIT Press: Southampton, 2000.

[11] Arslan, S. \& Sorguc A.G., Similarities between "structures in nature" and "man-made structures": biomimesis in architecture. Design and Nature II, WIT Press: Southampton, 2004.

[12] Jurkauskas, A. \& Susniene, D., Public transport in the sustainable urban transport policy. Urban Transport X, The Built Environment, Vol. 75, 2004.

[13] Wu, Q., Andreopoulos, Y. \& Weinbaum, S., Lessons learned from the exquisite design of the endothelial surface glycocalyx and their amazing applications. Design and Nature II, WIT Press: Southampton, 2004.

[14] Warren, L., Bound Carbonates in Nature, Cambridge University Press, 1996.

[15] Roelfsema, P.R., Engel, A.K., Konig, P. \& Singer, W., Visuomotor integration is associated with zero time-lag synchronization among cortical areas. Nature, 385, pp. 157-161, 1997.

[16] Kiuchi, T. \& Shireman, B., What We Learned in the Rainforest: Business Lessons From Nature, Future 500 Book, Berrett-Koehler: San Francisco, CA, 2004. 